adhesions. Thence it spread all over the abdomen. If this reasoning be correct, the interest lies in the extreme rapidity of the process; the whole course being completed within two months. Evidence of this remarkable acuteness lies in the complete absence of (1) enlarged glands, (2) abdominal adhesions, (3) any areas of caseation.

Passing on to the question of treatment I think one lesson stands out to be learnt from the results of removal of fluid.

The fluid thrown out may possibly be considered in the light of a safeguard designed by Nature as an artificial way of keeping the flaccid intestines floating out of each other's way. A warning of this possibility was given when, after the operation, symptoms of partial obstruction appeared. At the time 1 thought this due simply to paralysis of the gut, which would be accentuated by the dose of morphine, and the possible effect due to the removal of fluid did not assume the importance which I now give to it. The symptoms all became ameliorated as soon as the fluid had reaccumulated to any appreciable quantity, presumably as soon as the intestines were kept floating. On the other hand, when I carefully drained away all the fluid again by tapping, symptoms of obstruction reappeared, and this time progressed steadily and increasingly, giving evidence of actual kinks and not merely of paralysis. Undoubtedly, it was essential to relieve the tension in the peritoneal cavity, both because of the pressure on the heart and lungs and on the newly healed wound; but if the opportunity arose again I should be careful to remove enough only to relieve the tension, repeating the operation as often as necessary, rather than to entirely drain the cavity.

With regard to the effects of tuberculin, in such an acute case one could hardly expect much benefit from the treatment. As the index chart shows, the patient was constantly inoculating herself, and was possibly dealing, herself, with doses of tuberculin far larger than my own administrations.

It was impossible to say that at the actual moment of injection the blood had not been recharged in the interval that must necessarily have elapsed from the time of drawing the serum for the examination of the index; and though it was certainly unsafe to attempt larger doses for injection, it is doubtful whether such small quantities could be of any value.

One point of interest finally deserves mention, namely, the attack of heart failure which came on from thirty-six to forty hours after the operation. It was too late to be due to anaesthetic shock, and the question of its origin lies between a morphine toxaemia and some obscure circulatory affection directly concerned with the disease under consideration.

With regard to the morphine, opinions of course differ widely upon the propriety of ever giving morphine at all after abdominal operations. Every one will of course agree that it is a drug that should be avoided if possible, but where rest is especially indicated, as it appeared to me to be so in this instance, the benefit gained by it is often considerable, and in any case it seems hardly credible that so small a dose as $\frac{1}{6}$ grain could be responsible for such severe heart failure. Again, the patient's general condition did not suggest morphine toxaemia; the pupils, though small, reacted easily to light, and were certainly not "pinpoint," and her mental condition was perfectly clear.

On the other hand, it is very difficult to explain from a circulatory point of view. The heart itself was never dilated, nor did it ever show any sign of disease, so that the cause of failure was unlikely to be purely cardiac, but the possibility of pressure of paralysed and distended guts upon a weakened heart suggests itself, and is.perhaps the most probable explanation.

In conclusion, I have to thank Dr. Barnes and Dr. Hewetson for very much help and advice throughout the case, and Dr. Beatrice Webb for kindly estimating the opsonic indices.

A BAZAAR on behalf of the Edinburgh University Women Students' Union was held in Edinburgh during the three days November 19th, 20th, and 2lst. The hope was that the sum of $£ 5,000$ or more would be procured thereby. Unfortunately, from various causes, sóme unavoidable, some avoidable, the sum hoped for was not attained. Probably only $£ 1,500$ will be available.
A CASE OF ABDOMINAL, PELVIC, AND LABIAL TUMOUR.

BY JOHN A. C. MACEWEN, M.B., B.Sc., F.F.P.8.GLASG.

SURGEON TO THE ELDER COTTAGE HOSPITAL, GOVAN; ASSISTANT SURGEON, GLASGOW ROYAL INFIRMARY; SENIOR ASBISTANT TO PROFESSOR OF SURGELY, GLASGOW UNIVERSITY.

THe tumour in the case described below was very large; while extraperitoneal, it occupied a great part of the anterior portion of the abdominal cavity, and, further, extended down into the pelvis, in front of the bladder, and thence, through a large opening in the perineum, into the labium, which was greatly distended by it. On account of its size, unusual situation and extent, and physical characteristics, considerable difficulty was experienced in arriving at a definite diagnosis, and, in order to present these difficulties to the redder, the facts are recorded in their chronological order.

History.

A woman aged 49 was admitted to the Elder Hospital at the end of January, 1908, complaining of a swelling between the end of January, 1908, complaining of a swelling between the thighs, which rendered walking difficult. She stated that she first noticed a small swelling on the left thigh, close to Poupart's This swelling was soft, and easily reducible, and generally disThis swelling was soft, and easily reducible, and generally dis-
appeared on lying down. It gradually increased in size, occupying the labium more and more as it did so. The growth for many years was very slow, but during the four months prior to admission it had increased fully a third in size. At first it caused no inconvenienee, but about four years ago patient began to suffer from a sense of fullness in the vagina, and, later, considerable general discomfort, associated with the difficulty in walking already mentioned. During the last six months micturition had become troublesome, the patient feeling that there was some obstruction to the passage of urine, which necessitated the emptying of the bladder every two hours. No difficulty the emptying of the bladder every two hours. No difficulty was experienced with the bowels, which were perfectly regular, a laxative rarely being required. At no time had the patient suffered actual pain, and, apart from the condition complained of, she had always enjoyed excellent health. Menstrug

always been perfectly regular, normal, and painless.
The patient was married and had had nine children born at full term and two miscarriages, the latter occurring the one before and the other after the last full-term child. The labours were all easy, no instruments being required, and the miscarriages likewise caused the patient but little inconvenience. She first noticed the swelling in the groin shor ly after the last miscarriage.

Condition on Admission.

The urine was normal, specific gravity 1026 ; no pus, albumen, or sugar. While of normal height, the patient was exceedingly stout and heavy. The abdomen was rather flaccid and pendulous, particularly in the lateral regions, and appeared to be laden with adipose tissue. The lower limbs were particularly faden with adipose tissue. The lower limbs were particularly fat, the fat terminating rather abruptly at the ankles, and giving the appearance of oedema. That the swelling was not oedematous

pressure. left labium was enormously distended, forming a covering for a mass which occupied its whole origin from the pubes and perineum, and which extended fully 6 in. down between the thighs, the outer wall being composed of labial tissue, while the inner was partly labial and partly vaginal. The vessels on the surface were much dilated, and the mass was dull to percussion, fairly firm to the touch, and not adherent to the skin, which, however, was not quite freely movable over it.

The impression conveyed by the examination so far was that the mass was a fibro-cellular tumour of the labium, such as is occasionally met with growing to a very large size, so as to occsionally met with growing to a very large size, so as to extend even as far as the knees. The history given fy the patient, however, together with certain facts elicily incarcerated perineal hernia. When the patient coughed a certain impulse perineal hernia. When the patient coughed a certain impulse was transmitted to the mass, which, further, was partially the fingers some considerable distance into the pelvis by the side of the vagina. Full reduction of the swelling was impossible, but after the patient had been kept in bed for a few days, it was noticed that the swelling was smaller, softer, and more reducible than before. It was noticed, further, that the partial reduction was sometimes accompanied by a slight gurgling sound, which appeared to increase the probability of bowel constituting the contents. Abdominal palpation did not reveal anything definite, the wall being so bulky as to render accurate palpation very difficult.

\section{First Operation.}

It was decided that the swefling, whether tumour or bowel was not confined to the labium, and it was therefore determined to open the abdomen.

A vertical incision was accordingly made from below the umbilicus to the pubes, and attention was at once arrested by the fact that the abdominal wall, while fat, was not as fat as 
had been supposed. The incision was deepened between the recti, and then a layer which at first was taken for peritoneum but which later was seen to consist of transversalis fascia only, was picked up and divided, when it was found that tissue resembling bladder wall had been opened into. Although the patient had passed water immediately before the operation com menced, it was deemed advisable to pass a catheter, but only about an ounce of urine was drawn off As a further precantion about an ounce of une was drawn off. As a further precaution a fresh portion of supposed peritoneum was picked up, this time a little more than an inch below the umbilicus, but again the same tissue was opened into. It was therefore decided to explore this tissue carefully, and, on introducing the finge gently, it was found that a separation between it and the transversalis fascia was easily effected. The separation was accordingly continued until there was exposed to view a soft bulky, rather gelatincus mass, which both in size and contour somewhat resembled the distended bladder. To ensure no mistake being made, a sound was now introduced into the bladder, when it was found to pass to the right of and behind the tumour. With the sound in position it was easy to separate bladder and tumour. At this stage it was a question. whether this tumour had any connexion with the mass in the labium.

Once, however, this gelatinous and apparently cystic mass had been separated from the surrounding parts so that it could be lifted out of the abdomen, it was found to form only the upper end of a large tumour which extended deeply into the pelvis in front of and to the left of the bladder. The pelvic portion was cylindrical in section, and about 3 in. in diameter. It was composed of firm, white, and somewhat gelatinous tissue which stripped off in layers, making it rather difficult to determine how much was normal tissue and how much tumour there being no capsule. The relationship of the tumour to the bladder was so remarkable that, now the upper extremity could be drawn out of the abdomen, the opportunity was taken of exploring its other relations. It was found then that the tumour occupied not merely a large portion of the pelvis, pushing bladder and uterus backwards and to the side, but that it reached to within $1 \frac{1}{2} \mathrm{in}$. Of the umbilicus, pushing the peritoneum, which therefore had not been opened by the abdominal incision, upwards to that extent in front of it. It was also observed now, on pulling upon the abdominal mass, that the distended labium decreased in bulk, and this feature became more and more noticeable as the separation of the tumour from the surrounding structures was proceeded with. This separation became increasingly difficult as the deeper portions of the pelvis were reached, and while it had been hoped to remove the whole tumour through the abdomen by invaginating the labium, and thus avoid the necessity of a perineal wound, the pelvic portion of the tumour ultimately became detached from the labial. As the patient was rather exhausted it was determined to delay the final steps of the operation on the labial portion of the tumour; and, as there was general oozing from the raw surfaces, it was deemed advisable to pack the wound.

\section{Second Operation.}

Two weeks later, the patient having in the meantime regained her strength, and the raw surfaces being now covered with granulations, an anaesthetic was again administered, and an elliptical portion of the skin of the distended labium, together with the remainder of the labial portion of the tumour, was removed, care being taken while so doing not to injure the urethra or vagina, which, while not adherent, were close to the mass. In appearance and structure this portion resembled the stalked pelvic portion, and, after its removal, there was a wide communication between the labium and the abdomen above the pubes. The whole wound was now packed the packing being decreased as the wound filled up. The patient pale an uninterrupted recore made an uninterrupted recovery. One feature was the abdominal cavity, this being to a large extent completed within a few days of the first operation.

\section{Description of Tumour.}

The tumour thus removed might be roughly compared in shape to a mushroom, and consisted of three parts : an expanded abdominal portion which was soft and rather gelatinous, and in size and shape resembled the distended bladder-it gave the impression of being cystic, and was of a reddish-purple colour ; the stalked pelvic portion was firmer than the abdominal, but still gelatinous, and also sodden; it was cylindrical, about 6 in. long, and was much paler in colour than the abdominal portion; the labial portion closely resembled the pelvic portion in consistence and colour, but was bulbous in shape.

In order to ascertain the structure of the tumour it was frozen and cut in longitudinal strips. Contrary to expectation, no cysts were found, but the whole tumour consisted of a loose spongy were found, but the whole tumour consisted of a loose spongy network, somewhat resembling the structure of a loofah sponge. Microscopical sections were also prepared from various portions, and these showed the structure to be of the character of soft fibroma; it was extremely vascular, the larger vessels possessing remarkably thick walls, while numberless sma

The tumour was fully a foot in length, and weighed almost $3 \mathrm{lb}$.

The most striking point about this tumour was its resemblance, both in external appearance and physical characteristics, to a hernia. The earlier history of easy reduction, and disappearance on lying down, and the later of partial reduction, frequently accompanied by a slight gurgling sound, and the diminution in bulk when the patient was confined to bed, could hardly have been more typical of a partially incarcerated hernia.

Probably the tumour originated in the ischio-rectal fossa, and extended from there into the labium, and also upwards (perforating the levator ani and fascial layers) in front of the bladder, which was pushed backwards and to the side towards the abdomen. The abdominal portion appeared to be the most actively growing part of the tumour, but the recent rapid increase in the size of the labial swelling may point to activity at that end also, or may have been due simply to gravity acting upon the increased mass. On the other hand, the tumour may possibly have originated in the pelvis, and have reached the labium by pushing down the vaginal wall in front of it, and displacing the levator ani to the side.

The amount of space which the tumour was able to occupy, not only in the abdomen and pelvis, but also in its exit through the perineum to the labium, without causing any serious consequences, was also striking, as was, further, the readiness with which the displaced viscera, carrying before them the peritoneum, reoccupied the space of which they had so long been deprived.

\section{THREE CASES OF TROPHIC LESIONS OCCURRING SYMMETRICALLY ON THE EXTREMITIES.}

By W. SIDNEY SHEPPARD, B.A., M.B., B.Сh.CantaB., ACTING SENIOR MEDICAL OFFICER, SINGAPORE.

IN No. 2 of the new series of the Journal of the Malaya Branch of the British Medical Association, published in December, 1905, I called attention to " a hitherto undescribed form of superficial dermatitis probably trophic in origin," and briefly described a typical case of the disease. Since then I have met with several other cases of this disorder, three of which are at present under my observation. The following record of these cases may prove of interest :

CASE I

A Chinese food-seller, aged 23, was admitted to the gaol

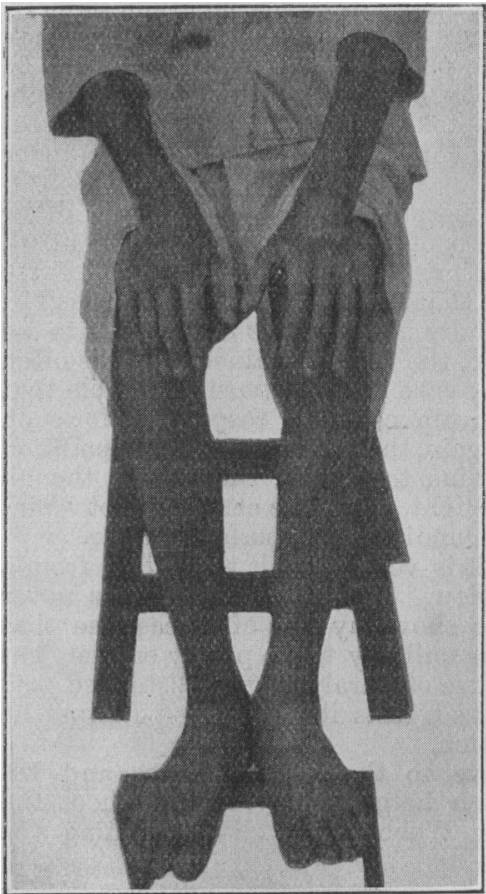
hospital on September 16 th.

$\mathrm{He}$ had syphilis three years ago. $\mathrm{He}$ was thin, of cachectic appearance; the nose had been partially destroyed; the thodestroyed; the thoorgans were healthy. organs were healthy. from albumen and sugar.

Occupying the dorsum of each foot, from the root of the toes to the front of the anklejoint, was a blister. The skin on the dorsum of the hands and on the distal two-thirds of the extensor surface of both forearms showed signs of recent blisters which had burst. The epidermis over the affected areas which had not been which had not been destroyed by the blisters was markedly to touch and temperature was lost over the whole area above described on both upper and lower limbs. Pain sensation was unaffected. The kneejerks were slightly exaggerated; there was no ankle clonus and no loss of motor power.

On October 8 th the whole of the affected areas were covered with healthy epidermis. On the upper limbs pigmentation was still very marked. Touch and temperature sensations were still in abeyance over the parts before mentioned, and also over the extensor surface of the legs. The anaesthesia over the legs may have been missed in my earlier examination. 